\title{
Observations
}

\section{Four Assumptions About Invariance in Perception}

\author{
James E. Cutting \\ Cornell University
}

\begin{abstract}
The term invariance has become more central to current views of perception. I take this as a good trend, but the term is rooted in mathematics, and its use in perception brings with it a host of assumptions that have generally been unexamined. The purpose of this article is to state some of these assumptions and assess their validity, with the hope that we can continue to find the term useful while acknowledging its limitations. The assumptions discussed are that (a) mathematics is an appropriate descriptive language for perception, (b) mathematical truths are transportable into perception without change of meaning, (c) mathematical imports are useful in explaining perception, and (d) perceptual invariants, like their mathematical counterparts, are absolute and not subject to threshold considerations.
\end{abstract}

If invariants of the energy flux at the receptors of an organism exist, and if these invariants correspond to the permanent properties of the environment, and if they are the basis of the organism's perception of the environment instead of the sensory data on which we have thought it based, then I think there is new support for realism in epistemology as well as for a new theory of perception in psychology. I may be wrong, but one way to find out is to submit this thesis to criticism. (Gibson, 1967, p. 162)

Heraclitus thought the world was ever changing; Parmenides thought it ever constant. In truth the world seems to be some of both. There are those things that change, sometimes called the variants, and those that do not, sometimes called invariants. From mathematics we get the idea that certain aspects of an object or event can be constant even while others are changing: Such things are said to be invariant under transformation. As suggested in the quote above, Gibson championed this idea within psychology, and particularly within visual perception. In recent years, this idea has seen increasing popularity, and there are, I think, good reasons for this upsurge. But underlying the cogent statement given above are many assumptions about invariance. In this article, I investigate four

This research was supported by National Institute of Mental Health Grant MH37467.

I thank William Epstein, Eleanor Gibson, Julian Hochberg, Carol Krumhansl, and Dennis Proffitt for their criticisms.

Requests for reprints should be sent to James E. Cutting, Department of Psychology, Uris Hall, Cornell University, Ithaca, New York 14853. of these. Since the term invariance is mathematical in origin, and since as psychologists we use it to huckster our ideas under the aegis of the Queen of the Sciences, the four assumptions discussed here deal with the intersection of mathematics and perception.

The current importance of the concept of invariance in perception is, of course, due to Gibson. The concept's use in perceptual theory, however, is much older. Consider, for example, the following passage from Helmholtz in his work, The Facts of Perception (1878/1971):

I should like, now, to return to the discussion of the most fundamental facts of perception. As we have seen, we not only have changing sense impressions which come to us without our doing anything; we also perceive while we are being active or moving about. ... Each movement we make by which we alter the appearance of objects should be thought of as an experiment designed to test whether we have understood correctly the invariant relations of the phenomena before us, that is, their existence in definite spatial relations.' (p. 384)

\footnotetext{
${ }^{1}$ The appearance of the term invariant here is due in part to translation. The same phrase is translated in Cohen and Wartofsky (1977, p. 136) as "lawlike behavior." In a similar manner, Cassirer $(1938 / 1944$, p. 10 ; translated by Gurwitsch) quoted Katz as follows: "The idea of invariance, which is an epistemological problem of validity of the foremost importance, has one of its roots, and perhaps the most nutritive one, in the psychology of perception." But in a different translation of Katz (1935, p. 185; translated by MacLeod and Fox), the same statement appears as follows: "The concept of 'constancy,' which involves an epistemological problem
} 
Although Helmholtz said much more than this, here at least he has clearly promoted the idea of an active organism exploring the invariants of an object or event undergoing transformations caused by exploration. That the notion of invariants was crucial to Helmholtz has not been lost to students of perception (Baird, 1970, p. 287; Cassirer, 1938/ 1944; Hochberg, 1974, p. 33, 1979, p. 103). In fact, Hochberg (1981b, p. 129) suggested that under normal conditions of perceiving, the positions of Helmholtz and Gibson are not distinguishable, and that Helmholtz and others, such as Mill, were very much concerned with invariants for perception:

Indeed, the idea of invariance appears in the tool chest of nearly every perceptual theorist. For example, Koffka used the term in many contexts. In Principles of Gestalt Psychology (Koffka, 1935), he quoted himself and analyzed the concept of invariance as he understood it:

"On the west side of Lake Cayuga, a couple of hundred feet or so above its level, stands a public building on a wide lawn that slants slightly towards the lake. To everyone this building seems to be tilted in a direction away from the lake in a most striking manner."

[Similarly] when we look through the window of [a] mountain railway carriage, this window becomes our spatial framework and appears, therefore, in normal horizontal-vertical orientation. The contours of the objects seen through the window do not intersect the sash at right angles. Therefore, if the sash is seen as horizontal, these objects cannot be seen as vertical. . . . If one sticks one's head out of the window, [a] telegraph pole will soon look vertical; when then, without losing sight of it, one withdraws the head, the telegraph pole will still appear vertical and the windows, the whole carriage, tilted. One factor in these two situations is invariant, the angle between ground and object.

It is easy to apply the same principle to the house on the western shores of Cayuga waters. The big lawn here provides the base, and therefore looks level. Consequently the house upon it must appear tilted. (pp. 217-218)

In other words, the ground is seen as invariably horizontal, and the building therefore appears tilted. Two things are important here. First, Koffka (1935, pp. 75-105) had a strong influence on Gibson, particularly in his question "Why do things look as they do?" (see Gibson, 1950, chap. 1; 1971). Second, Koffka used invariance somewhat differently than Helmholtz and Gibson did. Gone is the idea that aspects of the environment are invariant under transformation. Invariants for

of the greatest importance, has perhaps its most important root in the psychology of perception." What should be clear, then, is that the concept of invariance has been around a long time, but that the particular term is subject to the problems of translation.
Koffka are constancies without any particular mathematical implication. ${ }^{2}$

Gibson (1967), who was quoted in the introduction to this article, suggested that the concept of invariance in perception necessarily brings forth a new theory of perception. What is clear, however, is that this theory of perception is not wholly new. What is new to Gibson is the full emphasis on perceptual invariants, and the deemphasis on conceptual elaboration. Such a view contravenes Helmholtz's unconscious inference and Koffka's $(1935$, p. 80$)$ analysis of the nonstructure preserving mapping from distal to proximal stimulus and from proximal stimulus to percept (see also Epstein, 1977; Hochberg, 1981a).

In his book, The Perception of the Visual World, Gibson (1950) introduced the concept of invariance that was to influence his later work. It is interesting that he used the concept very little in that work (pp. 153-154) and only when he discussed a particular invariant from projective geometrythe cross ratio. It was Boring (1952), in his "Visual Perception as Invariance," who picked this idea out of Gibson's (1950) work and emphasized its importance. Boring was strongly influenced by Stevens's (1951, pp. 19-21) discussion of invariance as an idea that is central to all scientific endeavor (see also Cohen, 1931; Wigner; 1967). Meanwhile, the concept of invariance appeared in the literature independent of Gibson (e.g., Allport, 1955, pp. 607, 657; Luchins \& Luchins, 1964). Gibson was working on the idea of invariants throughout that period (Gibson, 1958; Gibson, Olum, \& Rosenblatt, 1955), and the first fullscale treatments appeared shortly thereafter (Gibson, 1959, 1960). After this point, although much of the rest of Gibson's theory changed, his discussion of invariants generally did not. He stuck fairly closely to the idea from mathematics (see, for example, Gibson, 1979, p. 310). The question is, however, what assumptions are made when the term invariance is used in perception?

\section{Assumption 1: Mathematics Is an Appropriate Descriptive Language for Perception}

One assumption made by Gibson and by a myriad of students of perception since Herbart is

\footnotetext{
${ }^{2}$ As suggested in Footnote 1, the terms invariance and constancy are closely related. The idea of constancies was introduced into psychology by the Gestalt psychologists and thus is in some sense a newer construal of invariance. But, of course, the discussion of the relations among distal stimuli, proximal stimuli, and percepts (although these terms are due to Koffka) is as old as the discussion of perception (see Epstein, 1977). I will not discuss constancies per se, in part because their discussion is not predicated on mathematics.
} 
that the best descriptors of perception are those taken from mathematics. This assumption transcends goals of elegance, formalism, and precision, and seems to have as its basis two ideas: First, the spatial layout of our perceptual world is best described in some form of mathematics (typically Euclidean geometry or projective geometry); ${ }^{3} \mathrm{sec}$ ond, the human mind is attuned to that description, since it is attuned to the layout.

With regard to the mathematics of space and its role in scientific thought, much discussion stems from Helmholtz (1857/1962, Vol. 3), Kant $(1770 / 1929)$, and early 19 th century natural philosophy (see Kline, 1959, 1980). Helmholtz, for example, suggested that Euclidean space is familiar and comprehensible to us-and that the spaces of Lobatchevsky, Riemann, and others are unfamiliar and difficult to imagine-because our experience is so incontrovertibly Euclidean. If we lived in a different universe, or even in a different local environment, Helmholtz suggested that we might have developed non-Euclidean geometries before Euclidean, and we might perceive in a nonEuclidean manner. ${ }^{4}$ The Euclidean space that we live in is three dimensional and not of higher dimensionality (Shepard, 1981), and historically the central problem for space perception is how three dimensions are perceived by a retinally based system with only two dimensions. This problem has been thought to be resolved, in part, through appeal to projective geometry (Johansson, von Hofsten, \& Jansson, 1980) and motion.

With regard to the intersection of mathematics and the structure of the mind, the central idea is at least as old as Galileo's mathesis universalis. This Renaissance idea seems very modern and is echoed by Pylyshyn (1972). We, as scientists, believe that

the secrets of the universe (both physical and psychological) are, as Galileo said, "written in the language of mathematics." But this must not be misunderstood to mean that it is only accessible to a mathematician. Even less does it mean that everything of importance can be measured and subjected to calculation. It means that those aspects of the universe that are ultimately comprehensible to the human mind are comprehensible because one can see in them a structure that is essentially mathematical. (pp. 547-548)

Whereas Pylyshyn was more concerned with the relation of linguistic formalisms to language, his statement applies equally well to the relation of perceptual theory to perception. In essence, many of us believe that the secrets of the perception of layout and of the perception of objects and events are partly understood through the mathematics of how these things are arrayed before us and how they change when we or they move.

Together, then, these twin ideas-(a) the structure of the world as mathematical and (b) that structure being comprehensible because of its mathematical nature - form one assumptive basis for realism as an epistemological position. It is the promise of tractability in mathematical description of the optic array that makes realism tenable for many of us and that promotes the study of ecological optics. To say that mathematics is an appropriate descriptive system for perception is, however, a global statement. It makes no particular commitment to the type of mathematics that may be relevant to perception. Thus, I know few psychologists who would overtly disagree with this assumption; it is very weak. Mathematics is so varied that it is difficult to believe, a priori, that this assumption could be false.

\section{Assumption 2: Mathematical Truths Are Transportable into Perception Without Change of Meaning}

It is one thing to say that the world and our perception of it are essentially mathematical. It is quite another to say that the tools of a particular branch of mathematics can be safely transported out of a rigorous and tightly circumscribed domain into an entirely different, less rigorous, and comparatively disordered domain like visual perception. Cassirer $(1938 / 1944)$ noted this when discussing group theory and perception:

The precision of mathematical concepts rests upon their being confined to a definite sphere. They cannot, without logical prejudice, be extended beyond that sphere into other domains. (p. 11)

In other words, Cassirer warned us that importation of mathematical ideas into the realm of perception can be a problematic course of action.

\footnotetext{
${ }^{3}$ The etymology of the term geometry is, of course, "earth measure," reinforcing the tenure of the connection between the perception of physical space and its measurement.

${ }^{4}$ Helmholtz was somewhat more careful than this, but this general attribution to Helmholtz is commonly found (see, for example, Boring, 1950, p. 315). Helmholtz was careful to distinguish between physical geometry of the real world and pure geometry as a mathematical discipline. Euclidean geometry, of course, is part of the latter. One reason for this distinction was Berkeley's dismissal of "natural geometry" as an alliance of physically apparent space and Euclidean axioms (see Epstein, 1977, pp. 12-13). Helmholtz recognized the predicament of the mathematics of space and their relation to perception: There were many maths but one world. Kline $(1959,1980)$, among others, attributed the fall of mathematics from its epistemologically central role in the natural sciences in the 19th century to the fact that mathematics could offer up many more geometries than were physically apparent. It is this issue, and the Kantian idea that "all properties of space are borrowed only from external relations through experience" (Kant, 1770/ 1929, Section 15D), that Helmholtz was concerned with.
} 
The structure of a particular branch of mathematics may bear no resemblance to the structure of perception and, more particularly, the problems of a particular branch of mathematics may bear no resemblance to those of perception. The implication is that if any aspect of mathematics and perception are nonisomorphic when dealing with a particular problem, then the application of that aspect of mathematics to that perceptual problem will be misleading.

Cassirer (1938/1944, p. 19), however, went further and made the strong claim that the principles of invariance and groups are the basis of both perception and geometric thought. Others have made similar claims (see, for example, Piaget, 1970). According to Cassirer, one can, through the instrumentality of these concepts, bring mathematical and psychological thought together under a common denominator. This claim may be true, but it is only partly empirical. I think we do not yet know enough about the utility of invariants in perception to make an informed judgment about this.

What should be clear is that Assumption 2 is a stronger and more particular version of Assumption 1: A specific kind of mathematics is asked to work for a specific problem in perception-that of how we perceive constancies in the world. Thus, we must assess whether the term invariance means the same thing in mathematics as it has come to mean in perception. To do this, we need more background on the term's use in mathematics.

\section{Digression on Invariance, Transformations, and Groups in Mathematics}

Invariance is a term born of mid-19th century mathematical thought. The term was first used, according to Bell (1945), by Boole in 1841 and Cayley in 1845 in algebra; it was first used, according to Klein (1908/1939), by Sylvester in 1850 in the same field. As the use of the term developed and spread, invariance came to mean "anything which is left unaltered by a coordinate transformation" (Thomas, 1944, p. 7). Later in the 19th century, with the work of Lie and Klein, the words invariance and transformation became interlocked.

Psychologists have heard most about the early history of invariance in the context of Klein's "Erlanger Programm" of 1872 (Michaels \& Carello, 1981; Piaget, 1970; Shaw, McIntyre, \& Mace, 1974). This program set about to codify the various types of geometries by the different types of invariance they maintained under different transformations (Bell, 1945). Michaels and Carello (1981, pp. 30-36; see also Shaw \& Pittenger, 1977, pp. 114-116) presented a clear discussion of the relationship of Euclidean space (which has dis- tance invariant), to similarity space (which has invariant ratios of similitude - objects can be expanded or contracted without loss of shape), to affine space (which has invariant ratios of division-strains or shears of one axis with another preserve collinearities and proportionalities), to projective space (which preserves cross ratios of four collinear points). Euclidean space has invariants under translation, picture-plane rotations, and reflections; similarity space has invariants under expansion and contraction; affine space has invariants under stretching or compression of a single axis or rotations of one axis against the other (as in parallel projection); and projective space has invariants under rotations out of the picture plane (as in polar projection). Klein's program defined a geometry as a system of definitions and theorems that remain invariant under a given group of transformations.

If invariants are those things in a geometry that are unaltered by coordinate change, we need to know more about these transformations and how they form a group. The key concept here is group in its mathematical, but not commonsensical, meaning. There are four postulates of a group (Bell, 1945, pp. 215-216; see also Stevens, 1951, p. 18):

1. Closure: If $a$ and $b$ are members of a set of operations (transformations) then $a^{\circ} b$ is also a member of the set. In other words, the group is closed. ( ${ }^{\circ}$ denotes combination.)

2. Association: For any three operations, $\left(a^{0} b\right)^{\circ} c=a^{\circ}\left(b^{\circ} c\right)$. In other words, pairwise order of combination of a string of operations is irrelevant.

3. Identity: There is an operation $i$ such that $a^{\circ} i=i^{\circ} a=a$. In other words, the group includes a null operation.

4. Reversion: There is an operation $a^{\prime}$ such that $a^{i o} a=i$. In other words, the existence of one operation implies its reverse as another member of the group.

Now consider what one can do with a block of wood on a desk. One can push it to the right (Operation $a$ ) and push it backwards the same extent (Operation $b$ ). One can also push it diagonally at $45^{\circ}$ until it finally rests in the same place, $a^{\circ} b$. If Operation $c$ is turning the block over, one can move the block diagonally and then turn it over, $\left(a^{\circ} b\right)^{\circ} c$, or one can move it to the right, then move it back an equal amount while turning it over, $a^{\circ}\left(b^{\circ} c\right)$. And of course, one can do nothing, $i$, and do the opposite of $a ; b$, and $c$. In addition, if the operations are also commutative (a fifth postulate), $a^{\circ} b=b^{\circ} a$, then the group is Abelian; the group of operations listed above is such a group. In Euclidean space, a rigid object like our block of wood can be moved around without changing its shape. All possible motions (or transforma- 
tions) of this object form a continuous groupthe "group of displacements" (Piaget, 1970, p. 20)-that is infinitely dense in potential operations along its various dimensions. Helmholtz tried to use this idea in an account of the perception of objects in space, and Cassirer (1938/1944) developed it in an attempt to coordinate perception and geometry. It is this coordination, if possible, that legitimizes the importation of the terms invariance, transformation, and group into perception without changing their mathematical meaning.

\section{Assumption 3: Mathematical Imports Are Useful in Explaining Perception}

It is one thing to import a term successfully from a different discipline, but is yet another to make it work for you. As an entrée in this discussion, consider again Klein's program and its efficacy in the late 19th and early 20th centuries within mathematics. Basically two things happened: The program ultimately failed, and where some of its ideas were generalized, the results seemed trivial (Bell, 1945, pp. 443-446). With regard to the first point, many new geometries were developed that did not fit the program. In particular, the concept of space developed such that its intrinsic structure might be, but generally could not be, defined in terms of transformation groups. But more relevant to our third assumption is the matter of trivialization in the application of groups, invariance, and transformation. The Erlanger Programm flourished for a few decades, and its ideas were applied to nearly everything imaginable. This brought problems, as Bell (1945) noted:

The success of the Erlanger Programm was also partly responsible for another tendency that did mathematics no particular good. When it was shown that a certain theory satisfied the postulates of a group, it seems to have been assumed as a matter of course that the theory was thereby significantly advanced. To cite a trivial instance, when it is gravely announced that all of the rational integers form a group with respect to addition, common sense will not stand open mouthed in dumb admiration, but will demand, "What of it?" (p. 446).

Like the application of groups to rational integers, any particular application of invariance, transformations, and groups to perception may be trivial. To say that everything one can do to a block of wood that retains its invariant character satisfies the postulates of an Abelian group does not appear to elucidate a theory of the perception of the block or a theory of action with that block; it simply states the obvious in an obfuscating manner. More generally, even if Cassirer's (1938/1944) assumption of invariance, transformations, and groups as common denominators for perception and geometry were true, it is not necessarily important for perception, nor is the equation of the concept of symmetry with invariance (Weyl, 1952). Both concepts simply state that object identity is preserved. It should be evident that perception is rule governed and yields constancies, since it works flawlessly most of the time. We don't need group theory to know this.

Consider a case in which these concepts do not particularly help. At the heart of group theory is the null transformation, $i$. When applied to perception, all possible objects and events are invariant (or symmetrical) under the null transformation. But this truth does not seem informative with regard to discovering the nature of how we perceive: To say that objects and events are invariant under the null transformation seems as vacuous as it is pedantic. One might try to remove the null transformation from the group-in essence, stating that we perceive invariants as revealed under all non-null transformations-but then we no longer have a group: The identity and reversion postulates have been violated. Moreover, it is not simply the null transformation that is problematic. The null transformation is completely surrounded by infinitesimal transformations that are also likely to be useless to perception. I will return to this when discussing Assumption 4.

What is important here, I believe, is that once the notion of invariants under coordinate transformations is applied to perception, it is an empirical matter as to whether it will be useful to perceptual theory. The link begins as a codification that may be circularly rooted in geometry and perception (Cassirer, 1938/1944), but unless one can specify that some invariants are used in perception and some are not, or that some transformations preserve functional invariants for perception and some do not, the use of the concepts of group, transformation, and invariance in perception may be little more than whistling in the breeze. In other words, I doubt that perceptual theory will be advanced without a partial decoupling of perception from these mathematical ideas.

\section{Digression on the Overgeneralization of the Concept of Invariants in Perception}

In group theory the members of the group are transformations, all related by the postulates given earlier. These transformations are operators, and what they operate on is left unchanged after the operation, which can always be thought of as a change in coordinates. What is important here is that there are two species of mathematical entities: invariants and transformations. Recently, Shaw 
and Pittenger (1977; see also Michaels \& Carello, 1981) have called these two varieties of the same species, that is, invariants. Shaw and Pittenger (1977, p. 113), for example, spoke of transformations or symmetry operations as being transformational invariants, and what they operate on as being symmetries or structural invariants. In various places, I have parsed event structure in essentially this same way (Cutting, 1981, 1982; Cutting \& Proffitt, 1981). However, I now think that the term transformational invariant and its cognates are misleading; perhaps they are even oxymorons. My reasons for this center on the idea that if we can make the term invariant useful to psychology, we ought to try to do it without changing its mathematical meaning.

Invariants are invariants because they survive transformations unchanged. But transformations are not invariant simply because they are unchanged by the entities that they operate on or are unchanged by other transformations. In other words, there is an asymmetry between invariants and transformations: Transformations operate on both invariants and variants and are themselves changed by neither; invariants are operated on by transformations and are unchanged, whereas variants are operated on by those transformations and are changed. It does not seem in the nature of transformations for them to be variant or invariant in any mathematical sense; they simply are what they are-operations. Another way to look at this, provisionally accepting the application of the term invariant to an operator, is to suggest that the term transformational invariant is uninformative because there appears to be no such thing, mathematically speaking, as a transformational variant. Objects, for example, do not operate on transformations such that they could change them. Pushing a block to the left will not become pushing it to the right or turning it over due to the character of the block. ${ }^{5}$

The tendency toward overgeneralization of the term invariant is also occasionally found in Gibson. For example, Gibson (1972, p. 221) noted that "A great many properties of the array are lawfully or regularly variant with changing observation point and this means that in each case a property defined by the law is invariant." Ullman $(1980$, p. 378$)$ criticized this position as being too vague and broad to be helpful in an analysis of perception, and I agree with Ullman. If we are to make the term invariance useful to perception, we must guard against its overgeneralization.

\section{Assumption 4: Perceptual Invariants Are} Absolute, as in Mathematics

When discussing the third assumption, I pointed out that the null transformation was not psycho- logically useful. This transformation, however, is not a special case; it is simply the center of a region of transformations in a continuous group that are so small as to be undetectable by a perceiver. In other words, there are likely to be an infinite number of transformations of an object in three-dimensional Euclidean space that are too small to reveal any invariants that are not revealed under the null transformation. This point was made implicitly by Luchins and Luchins (1964), and explicitly by Hochberg (1981a, pp. 276-277; 1982), but it was made earlier by Cassirer $(1938 / 1944)$ in a statement that weakens his argument on the parallel between geometry and perception:

It goes without saying that this analogy between the formation of invariants in perception and in geometry ought not to make us overlook the thoroughgoing differences which are very important from the epistemological point of view. These differences may be characterized by an expression which Plato used to define the opposition of perception to thought. All perception is confined to "more or less." . . . Only approximative, not absolute déterminations are attainable in perception. This realization is never ideally complete, but always remains within certain limits. The fixation of these limits constitutes one of the most important tasks of psychological research. Beyond these limits there is no further "transformation." (p. 16)

What Hochberg and Cassirer have said is that there are some transformations that simply are not psychologically relevant; they are too small. Any invariant not revealed under the null transformation is not revealed by these transformations either. Thus, there is a nonisomorphism between group theory and perception: In mathematics, all transformations reveal invariants, whereas in perception it may often be the case that only those transformations that are sufficiently large reveal invariants. Without acknowledgment of threshold considerations, a theory of perception based on invariants is simply a stimulus theory without necessary relevance to the organism (Epstein \& Park, 1964; Freeman, 1965, 1966).

This is much more than a quibble. One must not assume that we are simply dealing with necessarily minute changes in optical flux. Threshold determination for perceptual invariants is an em-

\footnotetext{
${ }^{5}$ This asymmetry is to be contrasted with structural invariants and structural variants, since both can exist. Thus, the term structural invariant seems better than transformational invariant, although without the benefit of its paired member, it may be redundant. The term's use (see, e.g., Shaw \& Pittenger, 1977) seems not far removed from the meaning of structure invariance as used in measurement theory (Luce \& Krumhansl, in press; Pfanzagl, 1968).
} 
pirical question, and some thresholds may be so high as to render questionable any use of them at all. Consider two examples: One of the invariants of layout is the density gradient (Gibson, 1950, p. 138 ) in which optically random arrangements of objects increase in density with distance. Through experimentation, however, we now know that the human eye is not very sensitive to density gradients per se as yielding perception of layout in depth (Braunstein, 1976; Marr, 1982), despite the fact that it is difficult to imagine any aspect of our environment that is more ecologically prevalent. What the eye is sensitive to is size-shape invariants. A second example, an invariant revealed through motion, is the center of the optical flow pattern (Gibson, 1950, p. 127ff; 1966). This point is said to tell an observer where he or she is going. Yet again through experimentation we know that the flow of a surface toward which an observer is moving orthogonally does not reveal such a point to the human observer to greater accuracy than about $10^{\circ}$ of visual angle (Johnston, White, \& Cumming, 1973; Llewellyn, 1971; Regan \& Beverley, 1982; Cutting, Note 1). Again, it is difficult to imagine many aspects of our environment that are much more pervasive: The surfaces of walls and buildings are all around us as we move between them. It would seem, then, that a density gradient and the center of optic flow are two invariants for which the "more or less" of our perception is a necessary concern.

\section{Summary}

I have tried to explicate four assumptions that I think underlie the application of the term invariance to perception. I make no claim that these exhaust the assumptions, and I emphasize that they are not independent. Moreover, they focus only on the idea of invariance as imported from mathematics-whether it has survived importation into psychology, whether immigration authorities have forced it to change its meaning, and whether it is a useful citizen.

I believe the first assumption to be valid, perhaps only because it is almost completely nonrestrictive: Mathematics is an appropriate descriptive language for perception, if only because its formalisms can take on a nearly infinite variety of forms.

I believe that the second and third assumptions can be valid if one is careful. Certain mathematical truths (such as invariance under transformation) are transportable into perception without loss of meaning and they can be useful to us. If these assumptions are to remain valid, psychologists must guard against contamination of the transplanted idea. Careful specification of invariants can help retain the vigor of the term, and wariness about overgeneralizations of the term will keep it from speciating beyond recognition. The term invariance may take hold and grow in perception, and the term transformation may as well, but these terms do not guarantee that the term group will be useful just because it belongs to the same alien family. Group theory, I believe, is not easily applied to perception as more than an empty formalism.

I believe the fourth assumption to be false: Because of the "more or less" of perception, perceptual invariants cannot be absolute. This fact forces us to realize that complete parallels between geometry and perception are unattainable and that invariance may not always be able to do the yeomanly work in perception that many of us might want. At best, invariants appear to work for us as perceivers and work for us as psychologists only some of the time. The determination of how well they work for perceivers and psychologists is an empirical question that is not helped by philosophical speculation and pronouncement.

\section{Reference Note}

1. Cutting, J. E. Motion parallax and visual flow: How to determine direction of locomotion. Paper presented at the fourth meeting of the International Society of Ecological Psychology, Hartford, Conn., October 1982.

\section{References}

Allport, F. H. Theories of perception and the concept of structure. New York: Wiley, 1955.

Baird, J. C. Psychophysical analysis of visual space. New York: Pergamon Press, 1970.

Bell, E. T. The development of mathematics. New York: McGraw-Hill, 1945.

Boring, E. G. A history of experimental psychology (2nd ed.). New York: Appleton-Century-Crofts, 1950.

Boring, E. G. Visual perception as invariance. Psychological Review, 1952, 59, 141-148.

Braunstein, M. Depth perception through motion. New York: Academic Press, 1976.

Cassirer, E. The concept of group and the theory of perception. (A. Gurwitsch, trans.). Philosophy and Phenomenological Research, 1944, 5, 1-35. (Originally published, 1938.)

Cohen, M. R. Reason and nature. Glencoe, IIl.: Free Press, 1931.

Cohen, R. S., \& Wartofsky, M. W. (Eds.). Hermann von Helmholtz: Epistemological writings. Boston Studies in the Philosophy of Science (Vol. 37). Boston: Dordrecht Riedel, 1977.

Cutting, J. E. Six tenets for event perception. Cognition, 1981, 10, 71-78.

Cutting, J. E. Two ecological perspectives: Gibson vs. Shaw and Turvey. American Journal of Psychology, 1982, 95, 199-222.

Cutting, J. E., \& Proffitt, D. R. Gait perception as an example of how we may perceive events. In $R$. Walk \& H. L. Pick (Eds.), Intersensory perception and sensory integration. New York: Plenum Press, 1981.

Epstein, W. Historical introduction to the constancies. 
In W. Epstein (Ed.), Stability and constancy in visual perception. New York: Wiley, 1977.

Epstein, W., \& Park, J. Examination for Gibson's psychophysical hypothesis. Psychological Bulletin, 1964, 62, 180-196.

Freeman, R. B. Ecological optics and visual slant. Psychological Review, 1965, 72, 501-504.

Freeman, R. B. Optical texture versus retinal perspective: A reply to Flock. Psychological Review, 1966, 73, 365371.

Gibson, J. J. The perception of the visual world. Boston: Houghton Mifflin, 1950.

Gibson, J. J. Visually controlled locomotion and visual orientation in animals. British Journal of Psychology, $1958,49,182-194$.

Gibson, J. J. Perception as a function of stimulation. In S. Koch (Ed.), Psychology: A study of a science (Vol. 1). New York: McGraw-Hill, 1959.

Gibson, J. J. Information contained in light. Acta Psychologica, 1960, 17, 23-30.

Gibson, J. J. New reasons for realism. Synthese, 1967, 17, 162-172.

Gibson, J. J. The legacies of Koffka's Principles. Journal of the History of the Behavioral Sciences, 1971, 7, 39.

Gibson, J. J. A theory of direct visual perception. In J. R. Royce \& W. W. Rozeboom (Eds.), The psychology of knowing. New York: Gordon \& Breach, 1972.

Gibson, J. J. The ecological approach to visual perception. Boston: Houghton Mifflin, 1979.

Gibson, J. J., Olum, P., \& Rosenblatt, F. Parallax and perspective during aircraft landings. American Journal of Psychology, 1955, 67, 372-385.

Helmholtz, H. Treatise on physiological optics. New York: Dover, 1962. (Originally published in German in 1857.)

Helmholtz, H. The facts of perception. In R. Kahl (Ed. and trans.), Selected writings of Hermann von Helmholtz. Middletown, Conn.: Wesleyan University Press, 1971. (Reprinted from Die Tatsachen in der Wahrnehmung, 1878.)

Hochberg, J. Higher-order stimuli and interresponse coupling in the perception of the visual world. In R. B. MacLeod \& H. L. Pick (Eds.), Perception: Essays in honor of James J. Gibson. Ithaca, N.Y.: Cornell University Press, 1974.

Hochberg, J. Sensation and perception. In E. Hearst (Ed.), The first century of experimental psychology. Hillsdale N.J.: Erlbaum, 1979.

Hochberg, J. Levels of perceptual organization. In M. Kubovy \& J. R. Pomerantz (Eds.), Perceptual organization. Hillsdale N.J.: Erlbaum, 1981. (a)

Hochberg, J. On cognition in perception: Perceptual coupling and unconscious inference. Cognition, 1981, 10, 127-134. (b)

Hochberg, J. How big is a stimulus? In J. Beck (Ed.), Organizational representation in perception. Hillsdale, N.J.: Erlbaum, 1982.

Johansson, G., von Hofsten, C., \& Jansson, G. Event perception. Annual Review of Psychology, 1980, 31, 27-63.

Johnston, I. R., White, G. R., \& Cumming, R. W. The role of optical expansion patterns in locomotor control. American Journal of Psychology, 1973, 86, 311324.
Kant, I. On the form and principles of the sensible and intelligible worlds. In J. Handyside (Ed. and trans.), Kant's innaugural dissertation and early writings on space. Chicago: Open Court, 1929. (Originally published, 1770.)

Katz, D. The world of color. (R. B. MacLeod \& C. W. Fox, trans.). London: Kegan Paul, Trench \& Trubner, 1935.

Klein, F. Elementary methematics from an advanced standpoint. New York: MacMillan, 1939. (Originally published in German in 1908.)

Kline, M. Mathematics and the physical world. New York: Dover, 1959.

Kline, M. Mathematics: The loss of certainty. New York: Oxford University Press, 1980.

Koffka, K. Principles of Gestalt psychology, New York: Harcourt, Brace, 1935.

Llewellyn, K. R. Visual guidance of locomotion. Journal of Experimental Psychology, 1971, 91, 245-261.

Luce, R. D., \& Krumhansl, C. L. Measurement, scaling, and psychophysics. In R. C. Atkinson, R. J. Herrnstein, G. Lindzey, \& R. D. Luce (Eds.), Handbook of experimental psychology (2nd ed.). New York: Wiley, in press.

Luchins, A. S., \& Luchins, E. H. On the study of invariants. Journal of General Psychology, 1964, 70, 265-277.

Marr, D. Vision. San Francisco: Freeman, 1982.

Michaels, C. F., \& Carello, C. Direct perception. Englewood Cliffs, N.J.: Prentice-Hall, 1981.

Pfanzagl, J. Theory of measurement. New York: Wiley, 1968.

Piaget, J. Structuralism. New York: Basic Books, 1970.

Pylyshyn, Z. Competence and psychological reality. American Psychologist, 1972, 27, 546-552.

Regan, D., \& Beverley, K. I. How do we avoid confounding the direction we are looking and the direction we are moving? Science, 1982, 215, 194-196.

Shaw, R. E., McIntyre, M., \& Mace, W. The role of symmetry in event perception. In R. B. MacLeod \& H. L. Pick (Eds.), Perception: Essays in honor of James J. Gibson. Ithaca, N.Y.: Cornell University Press, 1974.

Shaw, R. E., \& Pittenger, J. B. Perceiving the face of change in changing faces: Implications for a theory of object perception. In R. E. Shaw \& J. Bransford (Eds.), Perceiving, acting, and knowing. Hillsdale, N.J.: Erlbaum, 1977.

Shepard, R. N. Psychophysical complementarity. In M. Kubovy \& J. Pomerantz (Eds.), Perceptual organization. Hillsdale: N.J.: Erlbaum, 1981.

Stevens, S. S. Mathematics, measurement, and psychophysics. In S. S. Stevens (Ed.), Handbook of experimental psychology (1st ed.). New York: Wiley, 1951.

Thomas, T. The concept of invariance in mathematics. Berkeley: University of California Press, 1944.

Ullman, S. Against direct perception. Brain and Behavioral Sciences, 1980, 3, 373-415.

Weyl, H. Symmetry. Princeton, N.J.: Princeton University Press, 1952.

Wigner, E. P. Symmetries and reflections. Bloomington: Indiana University Press, 1967.

Received July 26, 1982

Revision received October 12, 1982 